\title{
Epidemiological Characteristics and Drug Resistance Analysis of Cerebrospinal Fluid Microbial Infections in Wenzhou Area
}

\author{
Teng Zhou ${ }^{1,2, *}$ \\ Mengjiao Kuang ${ }^{1, *}$ \\ Shirui Huang' \\ Jizhen $\mathrm{Li}^{\prime}$ \\ Qipeng Xie $\mathbb{D}^{\prime}$
}

'Department of Laboratory Medicine, The Second Affiliated Hospital \& Yuying Children's Hospital of Wenzhou Medical University, Wenzhou, Zhejiang, 325027, People's Republic of China; ${ }^{2}$ The Second Clinical Medical College, Wenzhou Medical University, Wenzhou, Zhejiang, 325035, People's Republic of China

*These authors contributed equally to this work
Correspondence: Qipeng Xie

Department of Laboratory Medicine, The Second Affiliated Hospital \& Yuying

Children's Hospital of Wenzhou Medical

University, Wenzhou, Zhejiang, 325027,

People's Republic of China

Tel +86 I5I57787/59

Email pandon2002@I63.com
Objective: Central nervous system infections (CNSI) are serious diseases that endanger human health. Identifying pathogens and their susceptibility to antibiotics, and promptly using antibiotics under this guidance is essential for treatment. The purpose of this study is to investigate the pathogen characteristics of CNSI patients, which can help clinicians choose appropriate empiric antibiotic .

Methods: We retrospectively collected data on CNSI patients with cerebrospinal fluid (CSF) culture positive from 2012 to 2020, including demographic characteristics, laboratory data, pathogenic bacteria, and antimicrobial susceptibility test results.

Results: A total of 166 patients with 168 isolates out of 8188 patients were available for data analysis. Among the isolates, Gram-positive bacteria, Gram-negative bacteria and fungi accounted for $59.5 \%, 36.3 \%$, and $4.2 \%$, respectively. Among newborns, children under 12 , and patients over 12, the most isolated strains were Streptococcus agalactiae (24/46, 52.2\%), Streptococcus pneumoniae $(21 / 68,30.9 \%)$ and Staphylococcus epidermidis (10/54, 18.5\%), respectively. Streptococcus agalactiae is more sensitive to linezolid and vancomycin. Streptococcus pneumoniae is more sensitive to vancomycin. Staphylococcus epidermidis is more sensitive to clindamycin and rifampicin. The sugar content in the CSF of Gramnegative bacteria of children $\leq 12$ years old was significantly lower than that of Gram-positive bacteria $(\mathrm{P}<0.05)$.

Conclusion: We comprehensively studied the etiological characteristics and antimicrobial resistance patterns of positive cerebrospinal fluid cultures in Wenzhou City, Zhejiang Province from 2012 to 2020 , which can provide valuable strategies for preventing pathogens and improving evidence-based treatment.

Keywords: cerebrospinal fluid, bacterial culture, drug resistance, epidemiological characteristics

\section{Introduction}

Central nervous system infection is one of the common clinical infectious diseases, which are mostly caused by pathogens invading the brain and spinal cord. According to the different infection pathogens, they can be divided into bacteria, viruses, fungi, tuberculosis, parasites and other causes. Compared with viruses and other pathogens, bacteria cause more infections in the central nervous system. In addition, many cases of acute infection occur in a flash, with rapid progress and high mortality. ${ }^{1-3}$ Therefore, the correct diagnosis and prompt use of antimicrobial therapy are crucial to the prognosis. 
Cerebrospinal fluid (CSF) is a colorless and transparent liquid, filling with various cerebral ventricles, subarachnoid spaces and central canal of spinal cord, providing nutrients and signaling molecules and clearing out toxic by-products from the brain. ${ }^{4}$ When suffering from a series of serious central system diseases such as meningitis, central system infection and radicular lesion, the bacterial culture results of cerebrospinal fluid are positive. Therefore, the results of cerebrospinal fluid bacterial culture can provide a basis for early diagnosis of the disease.

However, in recent years, the epidemiological studies on positive cerebrospinal fluid bacterial culture in Wenzhou have not been well characterized. At the same time, there are few reports regarding the antibiotic resistance patterns of prevalent bacteria in this area. Therefore, we comprehensively studied the etiological characteristics and antimicrobial resistance patterns of positive cerebrospinal fluid cultures in Wenzhou City, Zhejiang Province, from 2012 to 2020, which can provide valuable strategies for preventing pathogens and improving evidence-based treatment.

\section{Objects and Methods}

\section{General Information}

A total of 8188 cerebrospinal fluid samples were collected in the Second Affiliated Hospital of Wenzhou Medical University from 2012 to 2020, of which 166 cases were positive. The study was approved by the Ethical Committee of the Second Affiliated Hospital of Wenzhou Medical University (Approval No: 2021-K-11-01). Written informed consent of patients under 18 years old was obtained from the parents or legal guardians, and this study was conducted in accordance with the Declaration of Helsinki.

\section{Cerebrospinal Fluid Collection and} Routine Detection

For children with clinically suspected central nervous system infection, 2-3 mL CSF was collected under strict sterile operation in three tubes, and sent for routine, biochemical and microbiological tests within 1 hour. The cerebrospinal fluid cells were counted by the Neubauer counting chamber, and the nucleated cells were classified by the Wright-Giemsa staining. The content of glucose and chloride in the cerebrospinal fluid was detected using a DRY-CHEM7000 biochemical analyzer. The protein content of Cerebrospinal fluid was detected using an
ADVIA2400 biochemical analyzer. The determination was carried out in strict accordance with the operation manual and SOP, and the internal quality control was within the acceptable range.

\section{Culture and Identification of Cerebrospinal Fluid}

The cerebrospinal fluid was centrifuged at $3000 \mathrm{r} / \mathrm{min}$ in the microbiology laboratory, and the supernatant was discarded. The sediment was inoculated on Columbia blood plate, chocolate plate, and sand-weak plate, respectively, and incubated in an incubator for 24-72 $\mathrm{h}$ to observe the results. If the sample was positive, the pathogenic bacteria were isolated and cultured. If negative, reported aseptic growth. The bacterial strains were isolated and cultured in accordance with the National Operating Procedures for Clinical Testing, and the bacteria were identified by MALDI BioTyper ${ }^{\mathbb{R}}$ (MBT) automatic rapid microbial mass spectrometer.

\section{Drug Sensitive Test}

Automated drug sensitivity system test: The minimum inhibitory concentration (MIC) was detected according to the operating requirements of VITEK 2 compact, WalkAway and fungal drug sensitivity and the matching drug sensitivity card. K-B disk diffusion method: The diameter of antibacterial ring was measured with vernier caliper, and the results were interpreted according to the interpretation standard. The standards were subject to the National Operating Procedures for Clinical Examination and the Standards for Antimicrobial Susceptibility Test issued by CLSI (Clinical and Laboratory Standards Institute).

\section{Statistical Analysis}

Data processing and analysis were performed using SPSS 26.0 (SPSS Inc., Chicago, IL, USA). If the measurement data obeyed the normal distribution, they were expressed in the form of mean \pm standard deviation. The difference between two groups was compared by $t$-test and the differences among three groups were compared by analysis of variance. If data did not obey the normal distribution, median and upper and lower quartiles were used for expression. The Mann-Whitney $U$-test was used to compare differences between two groups, and the KruskalWallis $H$-test was used to compare differences among three groups. The count data were expressed in terms of the number of cases and the composition ratio, and the 
Table I Positive Profile of Cerebrospinal Fluid Culture Results

\begin{tabular}{|l|c|c|c|}
\hline Character & rouping & Cases (n) & Ratio (\%) \\
\hline Gender & Male & 94 & 56.0 \\
& Female & 74 & 44.0 \\
\hline \multirow{2}{*}{ Age } & Newborn ( 28 days) & 46 & 27.4 \\
& $\leq 12$ years old & 68 & 40.5 \\
\hline \multirow{3}{*}{ Season } & $>12$ years old & 54 & 32.1 \\
& Spring (March-May) & 52 & 31.0 \\
& Summer (June-August) & 42 & 25.0 \\
& Autumn (September-November) & 35 & 20.8 \\
& Winter (December-February) & 39 & 23.2 \\
\hline
\end{tabular}

composition ratio of two or more groups was compared using the chi-squared test. $\mathrm{P}<0.05$ indicated that the difference was statistically significant.

\section{Results}

\section{Epidemiological Survey of Pathogens in Cerebrospinal Fluid from January 2012 to October 2020}

A total of 8188 cerebrospinal fluid samples were collected from patients in the Second Affiliated Hospital of Wenzhou Medical University in 9 years, of which 166 were positive. Two pathogens were simultaneously detected in two patient specimens, so a total of 168 pathogens were detected. Men accounted for $56 \%$ and women accounted for $44 \%$. The positive results of cerebrospinal fluid culture were mainly in children less than 12 years old, accounting for $40.5 \%$ of the culture positive results. Meanwhile, the positive results were the most in spring (March-May) (53 cases, 31\%) and the least in autumn (September-November) (35 cases, 20.8\%), as shown in Table 1. In recent years, the positive results of cerebrospinal fluid culture showed a fluctuating upward trend, 17 cases in 2012; 8 cases in 2013; 16 cases in 2014; 6 cases in 2015; 20 cases in 2016; 31 cases in 2017; 28 cases in 2018; 24 cases in 2019; 18 cases in 2020 (Figure 1). According to the distribution of clinical symptoms in patients with positive cerebrospinal fluid culture, we found that patients with meningitis had higher cerebrospinal fluid positive culture results (105/168), most of them had purulent meningitis (95/105), and a few were fungal meningitis, viral meningitis and other various meningitis;

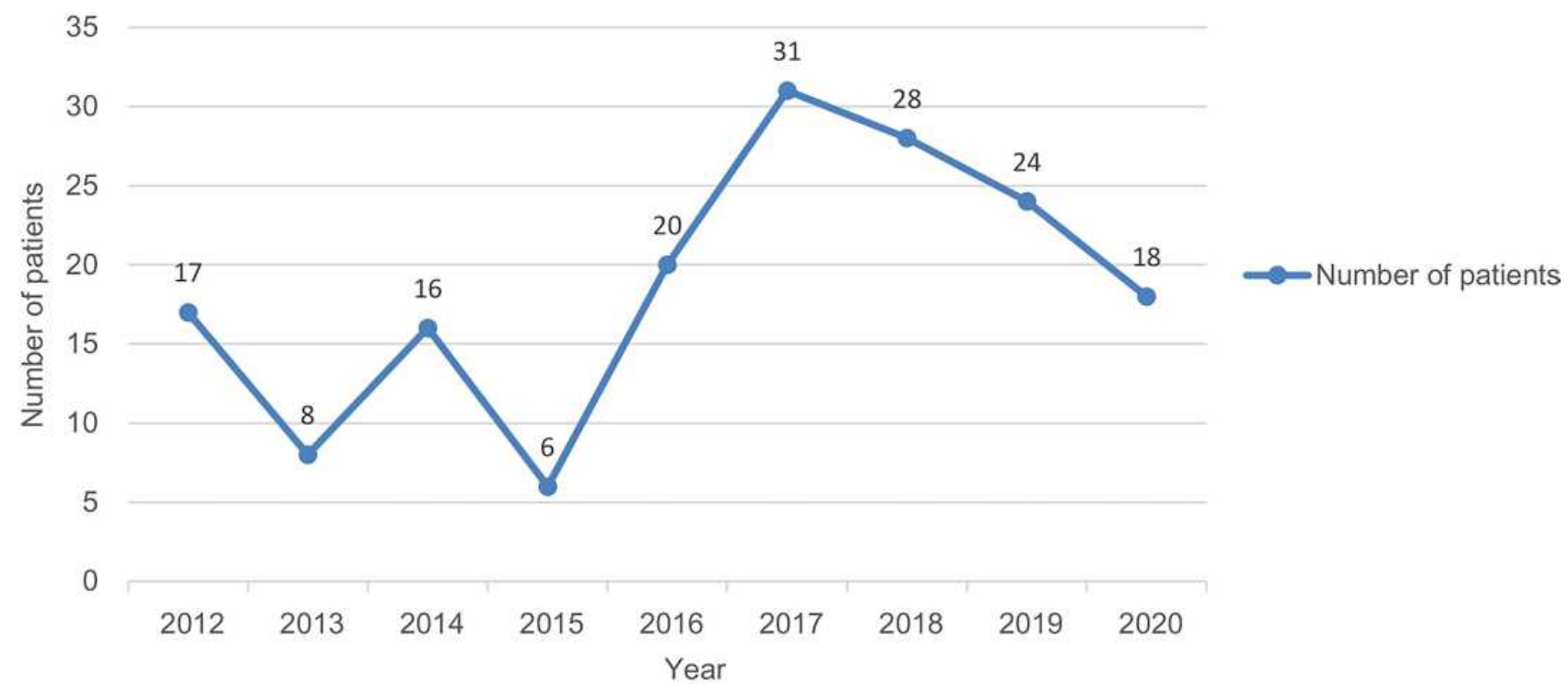

Figure I Time distribution diagram of culture positive result. 


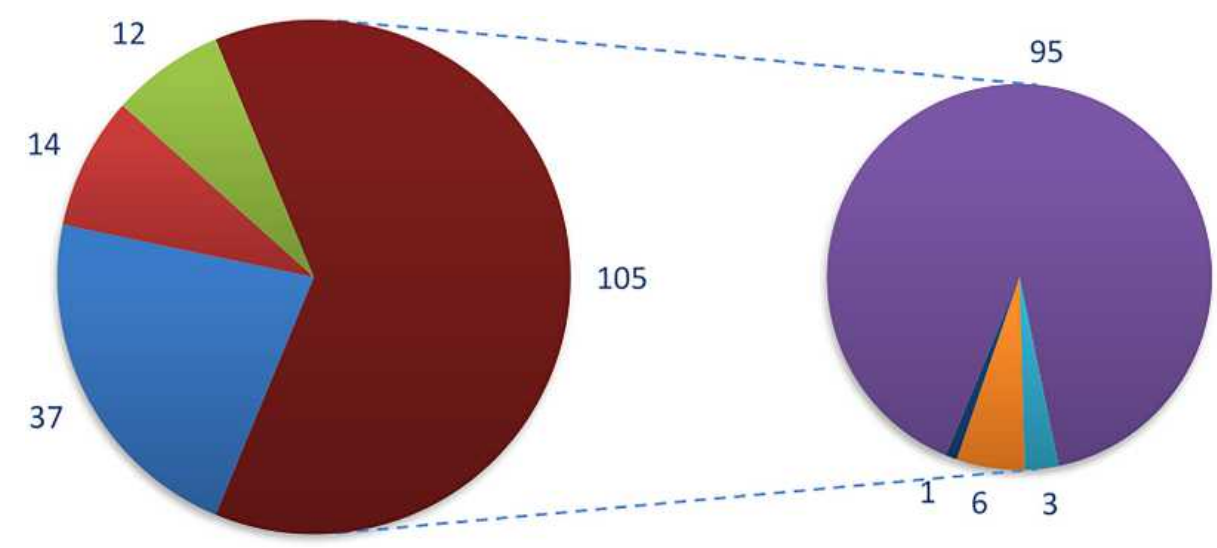

intracranial hemorrhage

hydrocephalus

other

Purulent meningitis

Viral meningitis

Fungal meningitis

tuberculous meningitis

Figure 2 Composite pie chart of clinical symptoms of patients with positive cerebrospinal fluid culture.

Meanwhile, positive cerebrospinal fluid culture may appear in symptoms such as intracranial hemorrhage and hydrocephalus (Figure 2).

The main prevalent microorganisms isolated in cerebrospinal fluid culture-positive specimens were Streptococcus agalactiae (19.0\%), Escherichia coli (13.7\%), Streptococcus pneumoniae (13.7\%), Staphylococcus epidermidis (13.1\%), and Klebsiella pneumoniae $(6.0 \%)$. In newborns ( $\leq 28$ days), Streptococcus agalactiae was the most commonly isolated bacterium from cerebrospinal fluid $(24 / 46,52.2 \%)$, followed by Escherichia coli (12/46, 26.1\%); However, in children under 12 years of age, the most common isolates were Streptococcus pneumoniae (21/68, 30.9\%), followed by Escherichia coli (10/68, 14.7\%), Staphylococcus epidermidis (9/68, 13.2\%), and Streptococcus agalactiae (8/68, $11.8 \%$ ); Furthermore, in patients older than 12 years, Staphylococcus epidermidis $(10 / 54,18.5 \%)$ was the most common, followed by Klebsiella pneumoniae (6/54, $11.1 \%)$. In addition, Cryptococcus neoformans $(3.6 \%)$ is the most common fungus isolated from culture-positive samples of cerebrospinal fluid, as shown in Table 2.

Further analysis of the significant differences in gender, age and season revealed that among G- bacteria, Escherichia coli was common under the age of 12 years, while Acinetobacter baumannii was common over the age of 12 years, and Klebsiella pneumoniae was common in autumn. For $\mathrm{G}^{+}$bacteria, more women than men had Streptococcus agalactiae infection, and Streptococcus agalactiae was common in newborns, Streptococcus pneumoniae was common in patients under 12 years of age, at the same time, Staphylococcus epidermidis infection was common in summer.

\section{Drug Resistance Analysis of Gram- Positive Pathogens in Cerebrospinal Fluid} The in vitro Gram-positive antimicrobial activities of the tested antibiotics are summarized in Table 3. Streptococcus agalactiae showed the highest sensitivity to linezolid and vancomycin, both $96.9 \%$, while it showed strong resistance to erythromycin, clindamycin and tetracycline, with the sensitivity rates of $6.3 \%, 6.3 \%$ and $3.1 \%$, respectively. Similar to Streptococcus agalactiae, Streptococcus pneumoniae also had the highest sensitivity to vancomycin, at $95.7 \%$, followed by chloramphenicol, moxifloxacin, ertapenem, and amoxicillin $(82.6 \%, 73.9 \%, 69.6 \%, 69.6 \%)$, and strong resistance to erythromycin, with the sensitivity rate of $4.3 \%$. Different from Streptococcus agalactiae, Staphylococcus epidermidis showed high sensitivity to clindamycin (90.9\%). At the same time, Staphylococcus epidermidis is also highly sensitive to rifampicin (86.4\%). However, it was not sensitive to penicillin, and the sensitivity rate was only $9.1 \%$.

\section{Drug Resistance Analysis of Gram- Negative Bacilli in Cerebrospinal Fluid}

The in vitro Gram-negative antimicrobial activity of the tested antibiotics is summarized in Table 4. Escherichia coli has a high sensitivity of $95.7 \%$ to amikacin, imipenem, and piperacillin/tazobactam, and a low sensitivity of $17.4 \%$ to ampicillin and ampicillin. Klebsiella pneumoniae was $80 \%$ sensitive to levofloxacin and trimethoprim-sulfamethoxazole and $0 \%$ sensitive to ampicillin. At the same time, it showed good sensitivity to certain $\beta$-lactams such as imipenem, piperacillin/tazobactam, cephalosporins, and aztreonam. 


\begin{tabular}{|c|c|c|c|c|c|c|c|c|c|c|c|c|c|c|c|c|c|c|c|c|c|c|}
\hline & 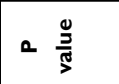 & $\begin{array}{l}+ \\
\stackrel{\$}{o} \\
0 \\
0\end{array}$ & 胥 & 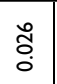 & बू. & $\stackrel{\infty}{\frac{\infty}{0}}$ & ' & 1 & 1 & 1 & 1 & 1 & 1 & 1 & 1 & 1 & 1 & 1 & 1 & 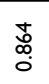 & হ̄ & 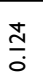 \\
\hline & 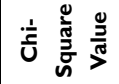 & 孛 & స్తి & $\underset{\substack { \infty \\
\begin{subarray}{c}{\infty \\
\sim{ \infty \\
\begin{subarray} { c } { \infty \\
\sim } }\end{subarray}}{ }$ & $\stackrel{\hat{~}}{\text { in }}$ & 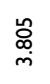 & ' & 1 & 1 & 1 & 1 & 1 & 1 & 1 & 1 & 1 & 1 & 1 & 1 & $\underset{\substack{\infty \\
\hat{0}}}{0}$ & $\underset{m}{\stackrel{9}{0}}$ & 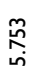 \\
\hline \multirow{4}{*}{ 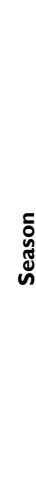 } & 离 & 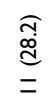 & $\begin{array}{l}\widehat{\widehat{m}} \\
\stackrel{\sigma}{\sigma}\end{array}$ & $\underset{\substack{\overrightarrow{0} \\
\sim}}{\bar{n}}$ & $\widehat{\overline{\vec{n}}}$ & 0 & 0 & 0 & 0 & 0 & 0 & o & 0 & 0 & $\circ$ & $\stackrel{\widehat{a}}{\stackrel{d}{-}}$ & 0 & 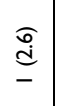 & $\stackrel{\widehat{d}}{\stackrel{d}{d}}$ &  & 命 & 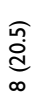 \\
\hline & 焉橧 & 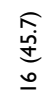 & $\stackrel{\substack{\infty \\
m}}{\infty}$ & 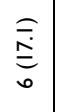 & 0 & 0 & $\underset{\substack{n \\
N}}{\tilde{\omega}}$ & 0 & $\underset{-}{\stackrel{\sigma}{\tilde{d}}}$ & $\underset{\widetilde{d}}{\underline{d}}$ & 0 & $\underset{-}{\stackrel{a}{d}}$ & $\underset{\text { de }}{\underline{d}}$ & 0 & 0 & $\circ$ & $\underset{-}{\stackrel{a}{d}}$ & 0 & 0 & $\begin{array}{l}\widehat{\widehat{m}} \\
\stackrel{f}{0} \\
\underline{\sigma} \\
\underline{a}\end{array}$ & $\underset{m}{\stackrel{\widehat{\infty}}{m}}$ & \\
\hline & 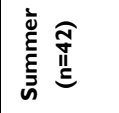 & 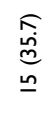 & 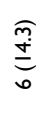 & 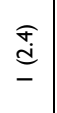 & $\underset{\substack{\text { d } \\
\sim}}{ }$ & $\overline{\bar{\Sigma}}$ & 0 & $\circ$ & $\underset{-}{\stackrel{\widetilde{d}}{\mathbb{d}}}$ & 0 & 0 & $\circ$ & 0 & $\underset{-}{\stackrel{f}{d}}$ & $\underset{d}{\stackrel{\mathbb{d}}{d}}$ & 0 & 0 & 0 & 0 & 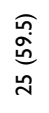 & $\underset{\infty}{\stackrel{a}{\sigma}}$ & 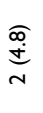 \\
\hline & 总 & 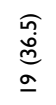 & $\begin{array}{l}\underset{\sigma}{\sigma} \\
\stackrel{0}{0} \\
\underline{0}\end{array}$ & $\stackrel{\sigma}{\stackrel{\sigma}{\Xi}}$ & $\begin{array}{l}\widehat{\widehat{D}} \\
\stackrel{\omega}{m} \\
m\end{array}$ & $\stackrel{\widehat{\infty}}{\stackrel{\sim}{\sim}}$ & $\circ$ & $\stackrel{\substack{\infty \\
\sim}}{\sim}$ & 0 & 0 & $\stackrel{\sigma}{\stackrel{\sigma}{\Xi}}$ & 0 & 0 & 0 & 0 & 0 & 0 & 0 & 0 & 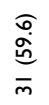 & $\begin{array}{l}\underset{\mathrm{o}}{\dot{d}} \\
\underline{m}\end{array}$ & 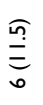 \\
\hline \multirow{5}{*}{  } & $2 \frac{\mathrm{O}}{\frac{2}{\nu}}$ & \begin{tabular}{|l|}
$\infty$ \\
$\stackrel{\infty}{0}$ \\
0
\end{tabular} & ั̊ & $\frac{\infty}{0}$ & 号 & 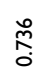 & ' & 1 & 1 & 1 & 1 & 1 & 1 & 1 & 1 & 1 & 1 & 1 & 1 & $\stackrel{\infty}{\circ}$ & o. & कृ \\
\hline & 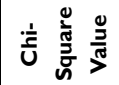 & 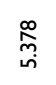 & $\begin{array}{l}\text { 主 } \\
\underline{\underline{y}}\end{array}$ & $\begin{array}{l}\bar{\jmath} \\
\bar{m}\end{array}$ & $\stackrel{i}{i}$ & 总 & ' & 1 & 1 & 1 & 1 & 1 & 1 & 1 & I & 1 & 1 & 1 & 1 & 节 & 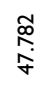 & 芯 \\
\hline & $\div \frac{\widetilde{f}}{\pi}$ & 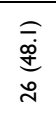 &  &  & $\widehat{\bar{\Xi}}$ & $\stackrel{\sigma}{\stackrel{\sigma}{E}}$ & $\underset{\substack{n \\
N}}{\mathbb{r}}$ & $\underset{\substack{i \\
\sim}}{\mathbb{n}}$ & $\underset{\substack{\tilde{j} \\
N}}{ }$ & $\circ$ & $\stackrel{\sigma}{\stackrel{\sigma}{=}}$ & $\stackrel{\sigma}{\stackrel{\sigma}{=}}$ & 0 & 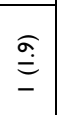 & o & $\circ$ & $\stackrel{\sigma}{\stackrel{\sigma}{E}}$ & $\stackrel{\sigma}{\stackrel{\sigma}{\Xi}}$ & $\stackrel{\sigma}{\stackrel{\sigma}{E}}$ & 总 & 。 & $\underset{\sim}{\stackrel{\Gamma}{\sim}}$ \\
\hline & viv & $\begin{array}{l}\sigma \\
\stackrel{a}{d} \\
d\end{array}$ & 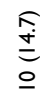 & $\underset{\sim}{\underset{d}{d}}$ & 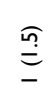 & $\underset{m}{\stackrel{\Im}{ \pm}}$ & 0 & $\circ$ & 0 & 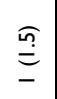 & 0 & $\circ$ & $\stackrel{\sqrt[n]{E}}{\underline{E}}$ & 0 & 0 & $\stackrel{\sqrt[n]{E}}{=}$ & 0 & 0 & 0 & $\begin{array}{l}\widehat{\bar{\theta}} \\
\hat{0}\end{array}$ & 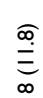 & 商 \\
\hline & 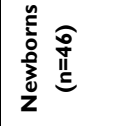 & 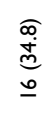 & $\underset{\overline{\vec{d}}}{\stackrel{\widehat{d}}{\underline{y}}}$ & 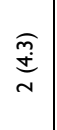 & 0 & $\underset{-}{\widetilde{d}}$ & $\circ$ & $\circ$ & 0 & 0 & $\circ$ & $\circ$ & $\circ$ & $\circ$ & $\underset{-}{\widetilde{d}}$ & 0 & $\circ$ & 0 & 0 & $\begin{array}{l}\text { तु } \\
\text { 苞 } \\
\text { o }\end{array}$ & 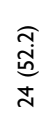 & 0 \\
\hline \multirow{4}{*}{ 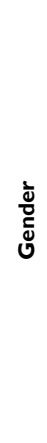 } & a. $\frac{\frac{0}{2}}{\frac{2}{2}}$ & 。 & 芯 & $\begin{array}{l}\stackrel{+}{+} \\
\stackrel{0}{0}\end{array}$ & $\underset{-}{0}$ & 峑 & 1 & 1 & 1 & 1 & 1 & 1 & 1 & 1 & 1 & 1 & 1 & I & 1 & ํㅗㅇ & : & $\frac{\pi}{0}$ \\
\hline & 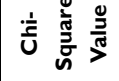 & ○ั & 范 & $\begin{array}{l}0 \\
: \\
0 \\
0\end{array}$ & ఫ̊ & 吢 & 1 & 1 & 1 & 1 & 1 & 1 & 1 & 1 & 1 & 1 & 1 & 1 & । & ఫ̊ & $\stackrel{\substack{+i}}{i}$ & 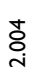 \\
\hline & 造 & $\begin{array}{l}\widehat{\widehat{n}} \\
\stackrel{0}{0} \\
\hat{n}\end{array}$ & $\begin{array}{l}\stackrel{\sqrt{n}}{\stackrel{m}{0}} \\
\underline{0}\end{array}$ & 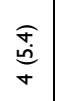 & $\overline{\dot{j}}$ & $\widehat{\overline{\dot{j}}}$ & 。 & $\underset{\sim}{\mathbb{d}}$ & 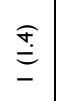 & 0 & $\stackrel{\overparen{f}}{\stackrel{\Xi}{E}}$ & 0 & $\stackrel{F}{\stackrel{F}{E}}$ & 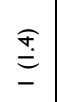 & $\stackrel{F}{\stackrel{F}{E}}$ & 0 & 0 & 0 & 0 & 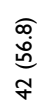 & 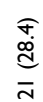 & $\frac{\sqrt[n]{a}}{2}$ \\
\hline & 造 & 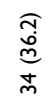 & $\underset{\substack{\infty \\
\stackrel{m}{=}}}{\underline{m}}$ & 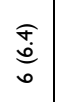 & $\widehat{\widehat{P}}$ & $\widehat{\overline{\mathrm{d}}}$ & $\widehat{\bar{d}}$ & 0 & $\stackrel{\widehat{\bar{\Xi}}}{\underline{\Xi}}$ & $\stackrel{\overline{\underline{\Xi}}}{\underline{-}}$ & 0 & 宔 & $\circ$ & 0 & 0 & $\stackrel{\overline{\dot{\Xi}}}{-}$ & $\stackrel{\bar{\Xi}}{\underline{E}}$ & $\stackrel{\widehat{\Xi}}{\underline{\Xi}}$ & $\stackrel{\overline{\dot{E}}}{\underline{-}}$ & 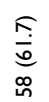 & $\begin{array}{l}\stackrel{\Gamma}{E} \\
=\end{array}$ & 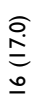 \\
\hline \multicolumn{2}{|c|}{ 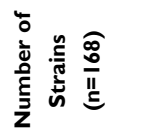 } & $\begin{array}{l}\widehat{\widehat{m}} \\
\stackrel{0}{0} \\
\overline{0} \\
\overline{0}\end{array}$ & 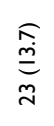 & $\begin{array}{l}\hat{a} \\
\dot{e} \\
\underline{0}\end{array}$ & 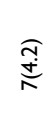 & 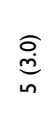 &  &  & 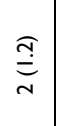 & 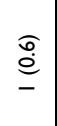 & $\begin{array}{l}\stackrel{0}{0} \\
\stackrel{-}{0}\end{array}$ & $\stackrel{\widehat{o}}{\stackrel{0}{0}}$ & $\underset{\stackrel{0}{\dot{e}}}{\stackrel{-}{-}}$ & $\stackrel{\widehat{o}}{\stackrel{0}{0}}$ & 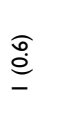 & $\stackrel{\widehat{o}}{\stackrel{\dot{\rho}}{-}}$ & $\stackrel{\widehat{0}}{\stackrel{0}{0}}$ & $\begin{array}{l}\stackrel{0}{\stackrel{0}{e}} \\
-\end{array}$ & $\stackrel{\widehat{o}}{\stackrel{0}{e}}$ & $\begin{array}{l}\widehat{\Omega} \\
\text { bn } \\
\text { 。 }\end{array}$ & 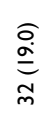 & 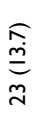 \\
\hline \multicolumn{2}{|l|}{ 总 } & 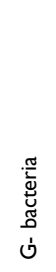 &  & 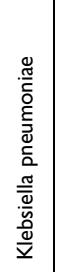 & 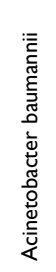 & 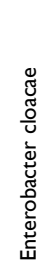 & 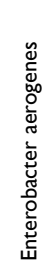 & 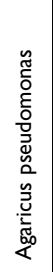 &  & 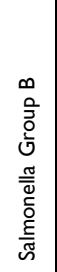 & 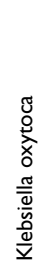 & 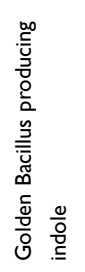 & 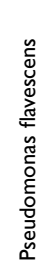 & 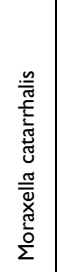 & 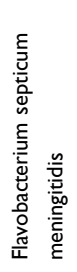 & 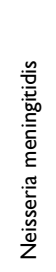 &  &  & 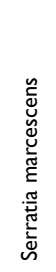 & 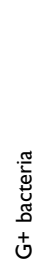 & 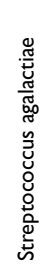 & 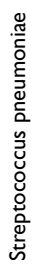 \\
\hline
\end{tabular}




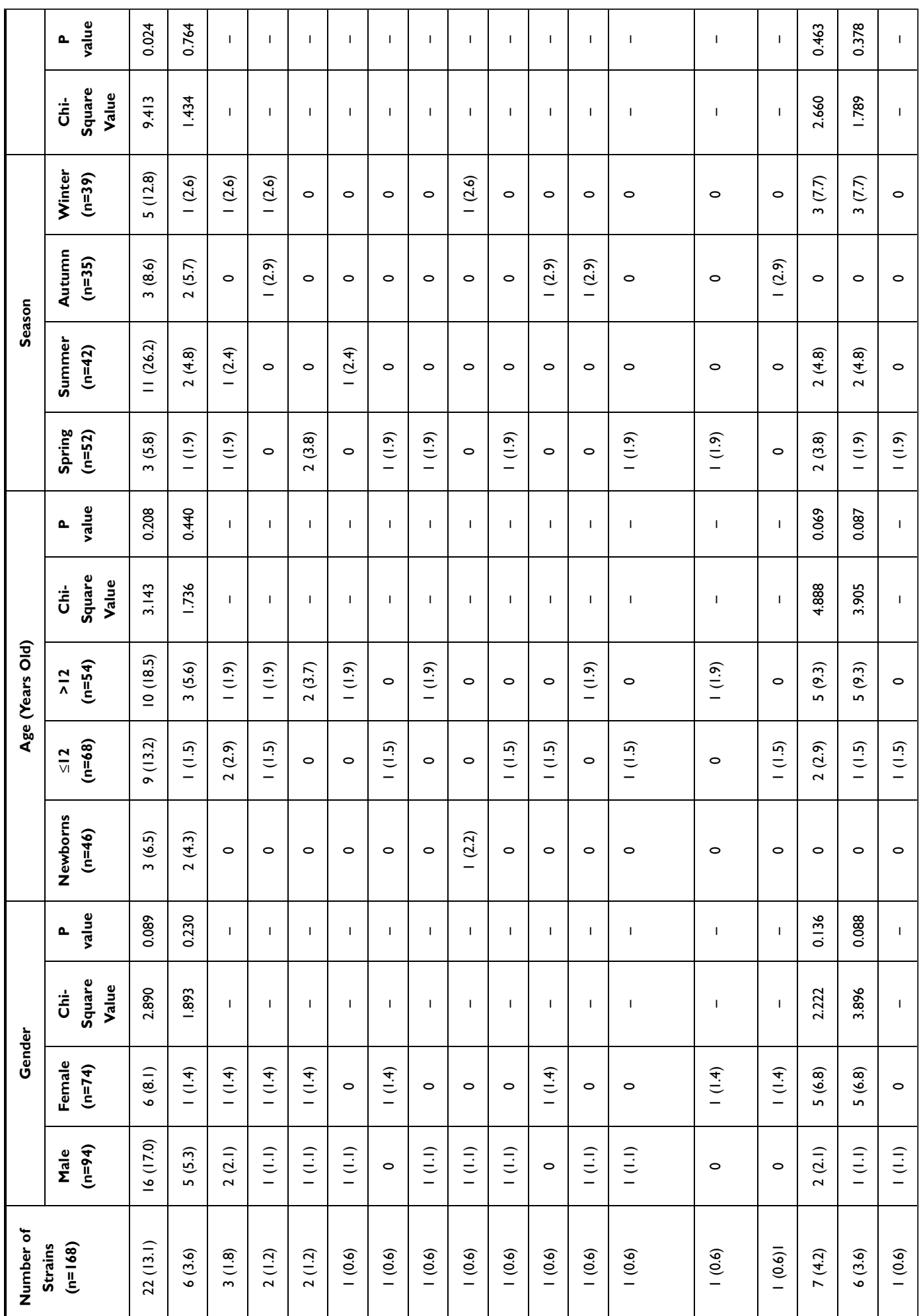

\begin{tabular}{|c|c|c|c|c|c|c|c|c|c|c|c|c|c|c|c|c|c|c|}
\hline 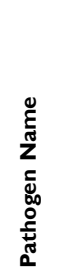 & 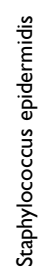 & 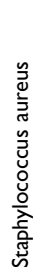 & 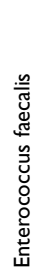 & 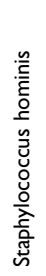 & 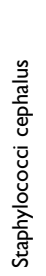 & 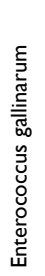 & 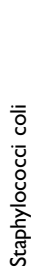 & 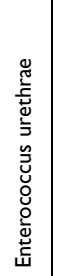 & 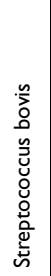 & 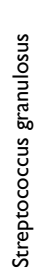 & 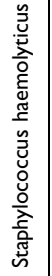 & 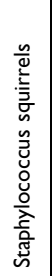 & 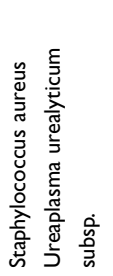 & 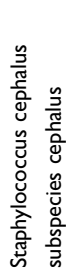 & 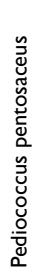 & 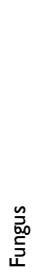 & 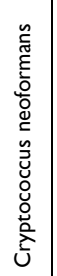 &  \\
\hline
\end{tabular}



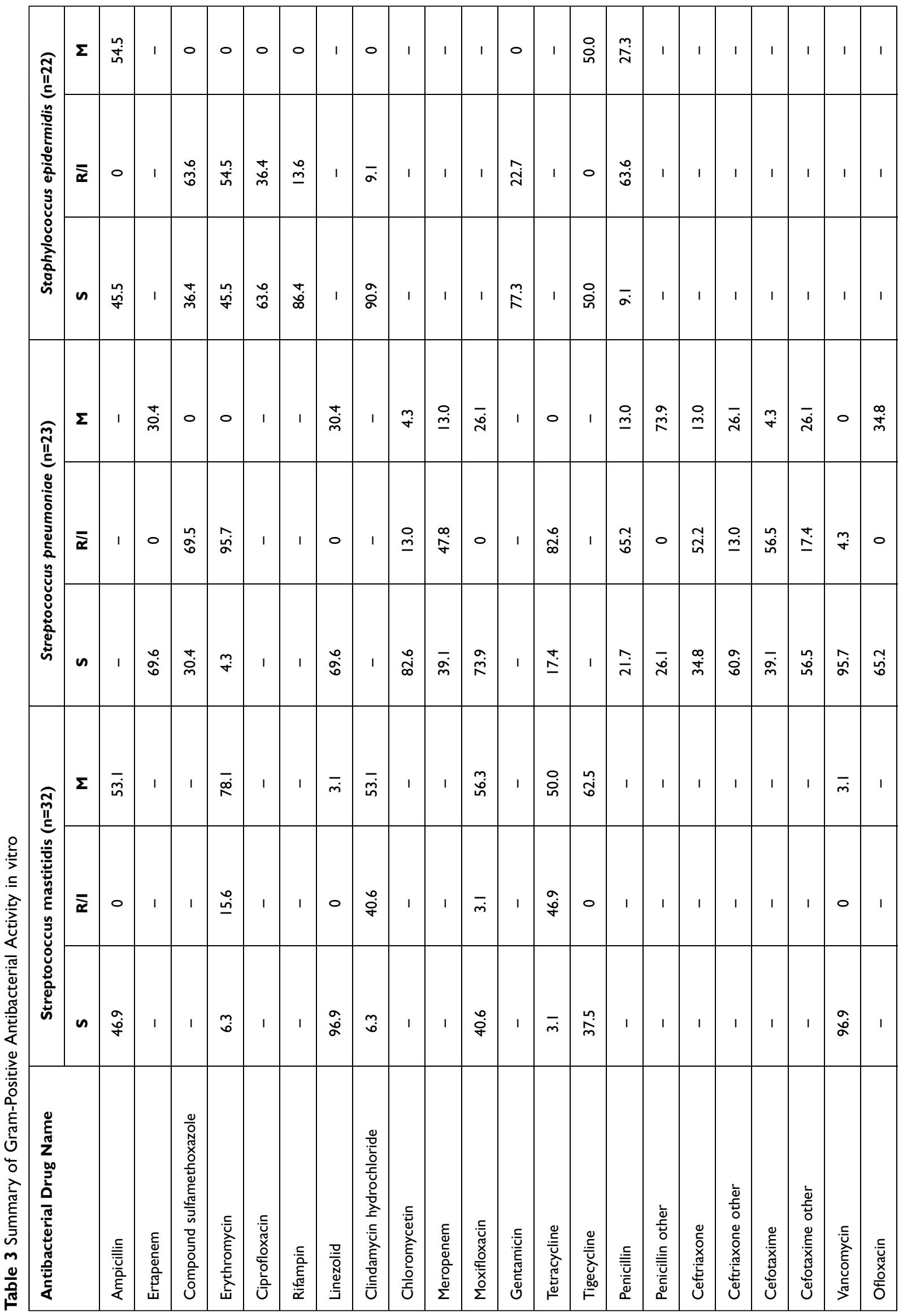


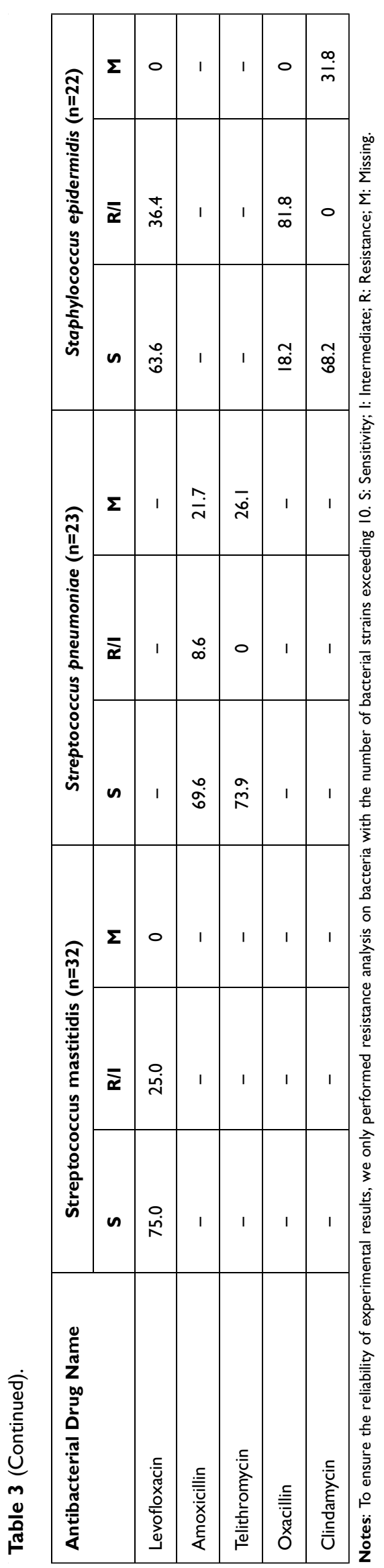

\section{Biochemical Detection of Positive Results of Cerebrospinal Fluid Culture}

The biochemical indicators such as white blood cell count $\left(10^{* 6}\right)$, red blood cell count $\left(10^{* 6}\right)$, neutrophil ratio, lymphocyte ratio, cerebrospinal fluid glucose quantification, chloride and protein quantification in patients with positive culture results are summarized in Table 5. The results showed that there were no significant differences in white blood cells, red blood cell counts, protein counts, cerebrospinal fluid glucose counts, and chloride counts between G- bacteria-infected and $\mathrm{G}+$ bacteria-infected CSF.

Through the analysis of patients in different age groups, we found that there was no significant difference in the above indicators of G- and G+ bacteria for patients old than 12 years old. While for patients younger than 12 years old, there is a significant difference in cerebrospinal fluid glucose quantification $(\mathrm{p}<0.05)$ (Tables 6 and 7 ). Therefore, the determination of cerebrospinal fluid glucose quantification can be used as an auxiliary judgment for Gor $\mathrm{G}+$ bacterial infection in cerebrospinal fluid of children under 12 years old.

\section{Discussion}

Central nervous system infection is one of the common clinical infectious diseases, which is characterized by acute onset, rapid progress, and high mortality. ${ }^{1-3}$ Cerebrospinal fluid (CSF) is a vital liquid, providing nutrients and signaling molecules, and clearing out toxic by-products from the brain. ${ }^{4}$ When the bacterial culture in the cerebrospinal fluid is positive, the patient may have a range of serious central system diseases, including meningitis, central system infections, and nerve root disease.

With the enhancement of bacterial resistance, despite the availability of new and powerful antibiotics, the mortality associated with neurological diseases, such as acute bacterial meningitis (ranging from $16 \%$ to $32 \%),{ }^{5-9}$ central nervous system infection after surgery $(8 \%)^{10}$ remains very high in some developing countries. In addition, according to the positive results of cerebrospinal fluid culture in recent years (Figure 1), the number of cerebrospinal fluid microbial infections has been shown a fluctuating upward trend in recent years, so it's necessary to analyze the epidemiology and drug resistance of cerebrospinal fluid microbial infections.

In this study, Streptococcus agalactiae (19.0\%), Escherichia coli (13.7\%), Streptococcus pneumoniae (13.7\%), Staphylococcus epidermidis (13.1\%), and Klebsiella pneumoniae $(6.0 \%)$ were the main prevalent microorganisms 


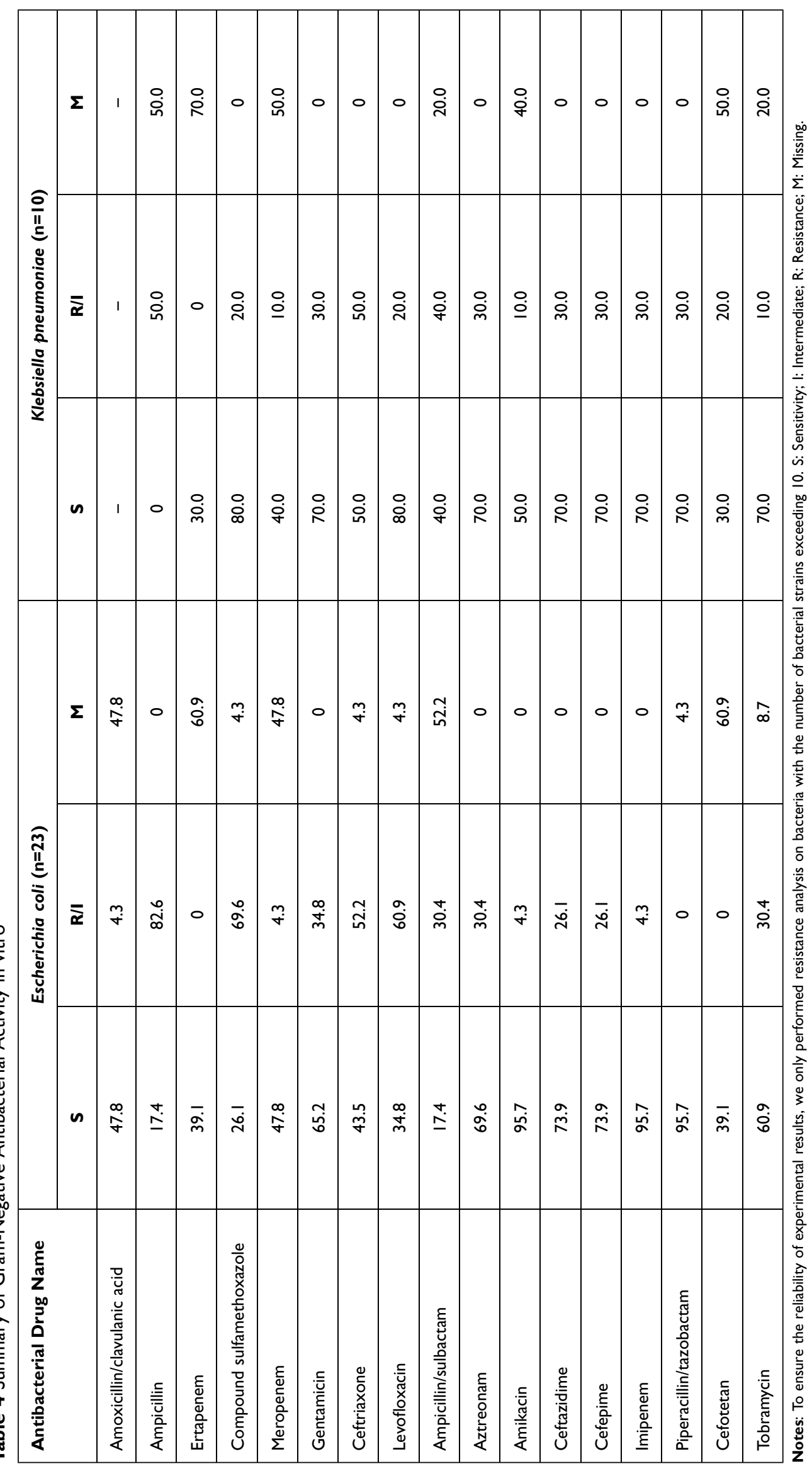


Table 5 Summary of CSF Index of Positive Culture Results

\begin{tabular}{|l|l|l|l|l|}
\hline Indicator & Gram - Bacteria & Gram + Bacteria & Statistics & P value \\
\hline White blood cell count $\left(10^{* 6}\right)$ & $1120.00(192.50,4130.00)$ & $680.00(144.00,2700.00)$ & -1.254 & 0.210 \\
\hline Red blood cell count $\left(10^{* 6}\right)$ & $97.50(10.00,752.50)$ & $90.00(9.00,565.00)$ & -0.019 & 0.985 \\
\hline Neutrophil ratio (\%) & $85(69,93)$ & $80(60,91)$ & -1.567 & 0.117 \\
\hline Lymphocyte ratio (\%) & $14(5,30)$ & $18(8,39)$ & -1.677 & -0.903 \\
\hline Glucose quantification (g/L) & $1.67 \pm 1.09$ & $1.83 \pm 1.15$ & -1.839 & 0.094 \\
\hline Chloride (mmol/L) & $113.47 \pm 8.82$ & $115.92 \pm 6.86$ & -0.203 & 0.069 \\
\hline Protein quantification (g/L) & $2.68(0.96,4.26)$ & $2.51(1.05,3.28)$ & 0.839 \\
\hline
\end{tabular}

Table 6 Association of G- and G+ Bacteria with CSF Indices in Children ( $\leq 12$ Years)

\begin{tabular}{|l|l|l|l|l|}
\hline Indicator & Gram - Bacteria & Gram + Bacteria & Statistics & P value \\
\hline White blood cell count $\left(10^{* 6}\right)$ & $1040.00(215.00,3400.00)$ & $645.00(152.25,3150.00)$ & -0.633 & 0.527 \\
\hline Red blood cell count $\left(10^{* 6}\right)$ & $84(68,90)$ & $79(60,92)$ & -0.377 & 0.706 \\
\hline Neutrophil ratio (\%) & $18(7,30)$ & $18(7,37)$ & -0.626 & 0.531 \\
\hline Lymphocyte ratio (\%) & $1.43 \pm 0.97$ & $1.70 \pm 1.09$ & -1.234 & 0.013 \\
\hline Glucose quantification (g/L) & $111.40 \pm 9.31$ & $115.21 \pm 6.24$ & -2.531 & 0.069 \\
\hline Chloride (mmol/L) & $2.49(1.03,4.33)$ & $2.76(1.00,3.28)$ & -0.033 & 0.974 \\
\hline
\end{tabular}

Table 7 Association of G- and G+ Bacteria with CSF Indices in Adult (>12 Years)

\begin{tabular}{|c|c|c|c|c|}
\hline Indicator & Gram - Bacteria & Gram + Bacteria & Statistics & $P$ value \\
\hline White blood cell count $\left(10^{* 6}\right)$ & $1200(185,557)$ & $750(101,1835)$ & -1.279 & 0.201 \\
\hline Red blood cell count $\left(10^{* 6}\right)$ & $89(71,96)$ & $82(54,88)$ & $-|.84|$ & 0.066 \\
\hline Neutrophil ratio (\%) & $10(4,27)$ & $18(10,52)$ & -1.749 & 0.080 \\
\hline Lymphocyte ratio (\%) & $1.97 \pm 1.18$ & $2.30 \pm 1.28$ & -0.935 & 0.354 \\
\hline Glucose quantification $(g / L)$ & $116.18 \pm 7.47$ & $118.41 \pm 8.39$ & -0.973 & 0.336 \\
\hline Chloride (mmol/L) & $2.74(0.94,3.16)$ & $1.74(1.09,3.12)$ & -0.549 & 0.583 \\
\hline
\end{tabular}

isolated from cerebrospinal fluid of Wenzhou patients from 2012 to 2020. Meanwhile, positive cerebrospinal fluid culture was most common in children less than 12 years old (including newborns), accounting for $67.9 \%$, and it was common in spring, which might be related to the suitability of microbial growth and reproduction in the warm environment of Wenzhou in spring. In addition, further analysis revealed that E. coli and Streptococcus pneumoniae were common under the age of 12 years, and Acinetobacter baumannii was common over the age of 12 years. Staphylococcus epidermidis infection in patients was more common in summer, and Klebsiella pneumoniae infection was more common in autumn, which had certain reference significance for doctors to assist in the diagnosis of neurological symptoms and empirical treatment in patients. At the same time, Streptococcus agalactiae infection affects more women than men and is common in newborns. This is highly consistent with some studies showing that women are the only clear risk factor associated with 
Streptococcus agalactiae meningitis. Therefore, clinicians should pay adequate attention to the presence of Streptococcus agalactiae infection in female infants younger than eight months at birth. ${ }^{11}$

Our study identified Streptococcus agalactiae as the most prevalent pathogen, which was identified in $19.0 \%$ of isolates obtained from the patients included in this study. In addition, Streptococcus agalactiae is the most frequently isolated bacteria from cerebrospinal fluid in newborns (less than 28 days), which is consistent with the related research that Streptococcus agalactiae (GBS) disease is caused by ascending infection of settled mothers or transmission during vaginal delivery, ${ }^{12}$ and related research shows that the disease is more common in newborns whose mothers are not screened by GBS, and Intrapartum antibiotic prophylaxis (IAP) is an effective intervention measure to reduce the incidence of earlyonset disease (EOD). ${ }^{13-15}$ Therefore, at present, the preventive measures of Streptococcus agalactiae should include prenatal GBS screening for women and antibiotic prophylaxis before and after delivery. ${ }^{16-18}$ Children or newborns with infection can be treated with antibiotics such as linezolid and vancomycin, and avoid using erythromycin, clindamycin, and tetracycline because Streptococcus agalactiae has a high resistance to them.

Like the epidemic bacterial infection in developing countries pointed out in relevant reports, E. coli is also prevalent in Wenzhou (13.7\%). ${ }^{19}$ Amikacin, imipenem, and piperacillin/tazobactam can be used clinically, but ampicillin should be avoided.

The other most common bacteria in children under 12 years of age is Streptococcus pneumoniae (17.8\%), which may be caused by the endogenous transmission of Streptococcus pneumoniae from the nasopharynx to the meninges, especially in the dry season, the nasopharynx is prone to crack and damage, especially for children with immature nasopharynx. ${ }^{20}$ This is in line with the seasonal distribution of positive results of cerebrospinal fluid separation- most in spring, because spring is relatively dry, which provides the best conditions for destroying mucosal defenses, which then leads to an increase in the number of cases of Streptococcus pneumoniae infection. Considering the adverse effects of drugs, vancomycin or chloramphenicol can be used for severe patients with Streptococcus pneumoniae infection, and $\beta$-lactam antibiotics such as ertapenem and amoxicillin can be used as far as possible for mild patients owning to their efficacy and less adverse effects, and avoid the use of erythromycin for treatment. Staphylococcus epidermidis $(13.1 \%)$ is the most common bacteria in children over 12 years old. We think that clindamycin and rifampicin can be used for clinical treatment, but penicillin is not recommended for treatment. Another common bacterium is Klebsiella pneumoniae, which is highly sensitive to synthetic antibiotics-levofloxacin and compound sulfamethoxazole, both of which are $80 \%$, but highly resistant to ampicillin, with a sensitivity rate of 0 . At the same time, it is sensitive to some $\beta$-lactams such as imipenem, piperacillin/tazobactam, cephalosporins, and aztreonam. The most common fungus is Cryptococcus neoformans, a kind of opportunistic yeast, which exist all over the world and interacts with various organisms. In humans, it causes cryptococcosis, a deadly invasive fungal infection that represents approximately 220,000 cases worldwide each year. ${ }^{21}$ Therefore, it is necessary for emergency treatment if suffering from cryptococcal infection.

Based on the biochemical indexes of cerebrospinal fluid, we found that white blood cell count, red blood cell count, and protein quantitation in cerebrospinal fluid are increased in patients with positive culture results, which was due to inflammatory reaction caused by microbial infection, white blood cells were attracted by chemokines. At the same time, most patients with positive culture have symptoms of intracranial hemorrhage, so red blood cell count and protein quantitation increase. Some literatures show that there are statistical differences in cell count and protein quantitation between Gram-positive bacteria and Gram-negative bacteria $(\mathrm{P}<0.05) .{ }^{22}$ However, we only found a significant difference in sugar quantification between Gram-positive and Gram-negative bacteria in the cerebrospinal fluid of patients under 12 years of age $(\mathrm{P}<0.05)$, which is different from the previous results.

In conclusion, this study investigated the patients with positive culture of cerebrospinal fluid in Wenzhou, excluded the patients with negative culture results, and obtained the epidemiological characteristics and drug resistance of microbial infection in Wenzhou. Streptococcus agalactiae is common in newborns. Streptococcus pneumoniae is common in children under 12 years old. Staphylococcus epidermidis is common in patients over 12 years old. Streptococcus agalactiae has certain drug resistance to macrolides, tetracyclines and lincomycin, and is highly sensitive to vancomycin and linezolid. However, the latter two have serious adverse reactions. Linezolid may cause pseudomembranous colitis and optic neuropathy. ${ }^{23-25}$ Vancomycin has severe nephrotoxicity and ototoxicity. Unfortunately, these adverse reactions are more sensitive to newborns who are susceptible to Streptococcus agalactiae. Therefore, the prospect of treating Streptococcus agalactiae infection in Wenzhou is worrying. 
Therefore, there is an urgent need to produce new drugs for pregnant women and pay attention to IAP to reduce the infection of neonatal Streptococcus agalactiae. Similar to Streptococcus agalactiae, although Streptococcus pneumoniae has the highest sensitivity to vancomycin or chloramphenicol, they all have serious adverse reactions. Streptomycin can cause aplastic anemia, hepatotoxicity, gray infant syndrome, etc. ${ }^{26,27}$ so it is not recommended to use in clinical practice. At the same time, recent studies have shown that penicillin-resistant Streptococcus pneumoniae is a serious problem in the world, ${ }^{28,29}$ which is consistent with our research results. Except for Ertapenem and Amoxicillin, Streptococcus pneumoniae is highly resistant to most $\beta$-lactam drugs, which may be related to the excessive use of $\beta$ lactam antibiotics in the clinic. Considering that $\beta$ lactams has fewer adverse reactions, we believe that ertapenem and amoxicillin are more suitable for the treatment of mild Streptococcus pneumoniae infection. Furthermore, Staphylococcus epidermidis is sensitive to clindamycin and rifampicin, and its adverse reactions are relatively mild, ${ }^{30,31}$ so we believe that they can be used as the first choice for the treatment of Staphylococcus epidermidis.

Study limitations: Our research has potential limitations. Because the incidence of central system infection is relatively low, although we have collected eight years, the sample size is still small, which may affect the results and produce deviations.

\section{Abbreviations}

CNSI, central nervous system infections; CSF, cerebrospinal fluid; MBT, MALDI BioTyper ${ }^{\circledR}$; MIC, the minimum inhibitory concentration; CLSI, Clinical and Laboratory Standards Institute; GBS, Group B streptococcus/ Streptococcus agalactiae; IAP, intrapartum antibiotic prophylaxis; EOD, early-onset disease.

\section{Data Sharing Statement}

The datasets generated and analyzed during the current study are available from the corresponding author on reasonable request.

\section{Ethics Approval and Consent to Participate}

The study was approved by the Ethical Committee of the Second Affiliated Hospital of Wenzhou Medical University (Approval No: 2021-K-11-01). Written informed consent of patients under 18 years old was obtained from the parents or legal guardians, and this study was conducted in accordance with the Declaration of Helsinki.

\section{Acknowledgments}

The results published here are completely based on the data detected by the Microbiology Laboratory of the Clinical Laboratory Center. We thank them for their contributions.

\section{Author Contributions}

All authors are aware and agree to the content of the paper and to their being listed as an author on the manuscript. All authors made a significant contribution to the work reported, whether that is in the conception, study design, execution, acquisition of data, analysis and interpretation, or in all these areas; took part in drafting, revising or critically reviewing the article; gave final approval of the version to be published; have agreed on the journal to which the article has been submitted; and agreed to be accountable for all aspects of the work.

\section{Funding}

This work was partially supported by grants from the Natural Science Foundation of China (NSFC81601849), Zhejiang Provincial Medicine and Health Technology Project (2019RC217), Wenzhou Science and Technology Bureau (Y20180109).

\section{Disclosure}

Neither this paper nor any similar paper has been or will be submitted to or published in any other scientific journal. There is no conflict of interest or competing financial interests for all authors.

\section{References}

1. Brouwer MC, Tunkel AR, van de Beek D. Epidemiology, diagnosis, and antimicrobial treatment of acute bacterial meningitis. Clin Microbiol Rev. 2010;23(3):467-492. doi:10.1128/CMR.00070-09

2. Gonzalez-Granado LI. Acute bacterial meningitis. Lancet Infect Dis. 2010;10(9):596. doi:10.1016/S1473-3099(10)70184-5

3. Jiang $\mathrm{H}$, Su M, Kui L, et al. Prevalence and antibiotic resistance profiles of cerebrospinal fluid pathogens in children with acute bacterial meningitis in Yunnan province, China, 2012-2015. PLoS One. 2017;12(6):e0180161. doi:10.1371/journal.pone.0180161

4. Pellegrini L, Bonfio C, Chadwick J, et al. Human CNS barrier-forming organoids with cerebrospinal fluid production. Science. 2020;369 (6500):eaaz5626. doi:10.1126/science.aaz5626

5. Jarousha AM, Afifi AA. Epidemiology and risk factors associated with developing bacterial meningitis among children in gaza strip. Iran $J$ Public Health. 2014;43(9):1176-1183. 
6. Shrestha RG, Tandukar S, Ansari S, et al. Bacterial meningitis in children under 15 years of age in Nepal. BMC Pediatr. 2015;15 (1):94. doi:10.1186/s12887-015-0416-6

7. McCormick DW, Wilson ML, Mankhambo L, et al. Risk factors for death and severe sequelae in Malawian children with bacterial meningitis, 1997-2010. Pediatr Infect Dis J. 2013;32(2):e54-61. doi:10.1097/INF.0b013e31826faf5a

8. Namani S, Milenković Z, Kuchar E, et al. Mortality from bacterial meningitis in children in Kosovo. $J$ Child Neurol. 2012;27(1):46-50.

9. Nickerson JW, A Attaran, BD Westerberg, et al. Fatal bacterial meningitis possibly associated with substandard ceftriaxoneUganda, 2013. MMWR Morb Mortal Wkly Rep. 2016;64(5051):1375-1377.

10. Chang JB, Wu H, Wang $\mathrm{H}$, et al. Prevalence and antibiotic resistance of bacteria isolated from the cerebrospinal fluid of neurosurgical patients at Peking Union Medical College Hospital. Antimicrob Resist Infect Control. 2018;7(1):41. doi:10.1186/s13756-018-0323-3

11. Bartlett AW, Smith B, George CRR, et al. Epidemiology of late and very late onset group b streptococcal disease: fifteen-year experience from two Australian tertiary pediatric facilities. Pediatr Infect Dis J. 2017;36(1):20-24. doi:10.1097/INF.0000000000001345

12. Berardi A, Rossi C, Guidotti I, et al. Factors associated with intrapartum transmission of group B Streptococcus. Pediatr Infect Dis J. 2014;33(12):1211-1215. doi:10.1097/INF.0000000000000439

13. Al Luhidan L, Madani A, Albanyan EA, et al. Neonatal group B streptococcal infection in a tertiary care hospital in Saudi Arabia: a 13-year experience. Pediatr Infect Dis J. 2019;38(7):731-734. doi:10.1097/INF.0000000000002269

14. Preventing neonatal group B streptococcal infection. Intrapartum antibiotic prophylaxis in some high-risk situations. Prescrire Int. 2011;20(114):72-77.

15. Romain AS, Cohen R, Plainvert C, et al. Clinical and laboratory features of group $\mathrm{b}$ streptococcus meningitis in infants and newborns: study of 848 cases in France, 2001-2014. Clin Infect Dis. 2018;66 (6):857-864. doi:10.1093/cid/cix896

16. Verani JR, McGee L, Schrag SJ. Prevention of perinatal group B streptococcal disease-revised guidelines from CDC, 2010. MMWR Recomm Rep. 2010;59(Rr-10):1-36.

17. Cagno CK, Pettit JM, Weiss BD. Prevention of perinatal group B streptococcal disease: updated CDC guideline. Am Fam Physician. 2012;86(1):59-65.

18. Di Renzo GC, Melin P, Berardi A, et al. Intrapartum GBS screening and antibiotic prophylaxis: a European consensus conference. $J$ Matern Fetal Neonatal Med. 2015;28(7):766-782. doi:10.3109/ 14767058.2014.934804
19. Furyk JS, Swann O, Molyneux E. Systematic review: neonatal meningitis in the developing world. Trop Med Int Health. 2011;16 (6):672-679. doi:10.1111/j.1365-3156.2011.02750.x

20. Gervaix A, Taguebue J, Bescher BN, et al. Bacterial meningitis and pneumococcal serotype distribution in children in cameroon. Pediatr Infect Dis J. 2012;31(10):1084-1087. doi:10.1097/ INF.0b013e318260552d

21. Alanio A. Dormancy in Cryptococcus neoformans: 60 years of accumulating evidence. J Clin Invest. 2020;130(7):3353-3360. doi:10.1172/JCI136223

22. Ru L. 1861 cerebrospinal fluid test characteristics and clinical analysis (in Chinese). Pract J Clin Med. 2012;9(4):139-141.

23. Kishor K, Dhasmana N, Kamble S, et al. Linezolid induced adverse drug reactions - An update. Curr Drug Metab. 2015;16(7):553-559. doi:10.2174/1389200216666151001121004

24. Swaminathan A, Du Cros P, Seddon JA, et al. Peripheral neuropathy in a diabetic child treated with linezolid for multidrug-resistant tuberculosis: a case report and review of the literature. BMC Infect Dis. 2017;17(1):417. doi:10.1186/s12879-017-2499-1

25. Mehta S, M Das, C Laxmeshwar,et al. Linezolid-associated optic neuropathy in drug-resistant tuberculosis patients in Mumbai, India. PLoS One. 2016;11(9):e0162138. doi:10.1371/journal.pone.0162138

26. Li Y, Zhu Y, Zhong Q, et al. Serious adverse reactions from antituberculosis drugs among 599 children hospitalized for tuberculosis. Pediatr Infect Dis J. 2017;36(8):720-725. doi:10.1097/ INF.0000000000001532

27. Kargar M, Mansouri A, Hadjibabaie M, et al. Anti-tuberculosis drugs adverse reactions: a review of the Iranian literature. Expert Opin Drug Saf. 2014;13(7):875-891. doi:10.1517/14740338.2014.925443

28. Liu EY, Chang J-C, Lin J-C, et al. Important mutations contributing to high-level penicillin resistance in Taiwan 19F-14, Taiwan 23F-15, and Spain 23F-1 of Streptococcus pneumoniae Isolated from Taiwan. Microb Drug Resist. 2016;22(8):646-654. doi:10.1089/ mdr.2015.0261

29. Lu TL, Cao Y, Zhou L-X, et al. The antimicrobial resistance analysis of 73 strains of streptococcus pneumoniae isolated from infant respiratory tract. Sichuan Da Xue Xue Bao Yi Xue Ban. 2015;46 (3):417-421.

30. Klainer AS. Clindamycin. Med Clin North Am. 1987;71(6):11691175. doi:10.1016/S0025-7125(16)30804-5

31. Phillips I. Clinical uses and control of rifampicin and clindamycin. $J$ Clin Pathol. 1971;24(5):410-418. doi:10.1136/jcp.24.5.410
Infection and Drug Resistance

\section{Publish your work in this journal}

Infection and Drug Resistance is an international, peer-reviewed openaccess journal that focuses on the optimal treatment of infection (bacterial, fungal and viral) and the development and institution of preventive strategies to minimize the development and spread of resistance. The journal is specifically concerned with the epidemiology of antibiotic resistance and the mechanisms of resistance development and diffusion in both hospitals and the community. The manuscript management system is completely online and includes a very quick and fair peerreview system, which is all easy to use. Visit http://www.dovepress.com/ testimonials.php to read real quotes from published authors. 